\title{
Erratum: Quantitatively analyzing phonon spectral contribution of thermal conductivity based on nonequilibrium molecular dynamics simulations. II. From time Fourier transform [Phys. Rev. B 92, 195205 (2015)]
}

\author{
Yanguang Zhou and Ming $\mathrm{Hu}$ \\ (Received 22 December 2015; published 6 January 2016)
}

DOI: 10.1103/PhysRevB.93.039901

To help the readers more clearly understand the difference between the original paper and previous works, we would like to clarify that Eqs. (2)-(6) summarizing the derivation of the spectral decomposition of the heat current were originally derived in Ref. [1]. In Ref. [2], these equations were extended to calculate the spectral decomposition of thermal conductivity in carbon nanotubes. It is unfortunate that Ref. [2] only appeared in the Abstract of the original paper and not in the reference section too. Additionally, we point out that in order to unify the terminology in the first [3] and second parts of the original paper of our series papers, we refer to the spectral decomposition of the thermal conductivity method [2] as the frequency-domain direct decomposed method in our article.

[1] K. Sääskilahti, J. Oksanen, J. Tulkki, and S. Volz, Phys. Rev. B 90, 134312 (2014).

[2] K. Sääskilahti, J. Oksanen, S. Volz, and J. Tulkki, Phys. Rev. B 91, 115426 (2015).

[3] Y. Zhou, X. Zhang, and M. Hu, Phys. Rev. B 92, 195204 (2015). 